\title{
Some Results on the Krein Parameters of an Association Scheme
}

\author{
Vasco Moço Mano, Enide Andrade Martins and Luís Almeida Vieira
}

\begin{abstract}
We consider association schemes with $d$ classes and the underlying BoseMesner algebra, $\mathscr{A}$. Then, by taking into account the relationship between the Hadamard and the Kronecker products of matrices and making use of some matrix techniques over the idempotents of the unique basis of minimal orthogonal idempotents of $\mathscr{A}$, we prove some results over the Krein parameters of an association scheme.
\end{abstract}

\section{Introduction}

The concept of association scheme was defined by Bose and Shimamoto in 1952, [4], and constitutes a powerful algebra and combinatorics tool that has a wide range of applications from statistics, [2, 4], combinatorial designs, [2, 3, 4], coding theory, [6], group theory, [8, 9], or character theory, [7]. One can observe an association scheme with $d$ classes as a general and more complex combinatorial structure. In fact, each relation of an association scheme corresponds to an undirected graph and, as a particular example, an association scheme with just two classes is equivalent to a strongly regular graph and its complement.

Vasco Moço Mano

Faculty of Sciences of University of Porto, Rua do Campo Alegre, 687; 4169-007, Porto, Portugal e-mail: vascomocomano@gmail.com

Enide Andrade Martins

CIDMA-Center for Research and Development in Mathematics and Applications

Department of Mathematics, University of Aveiro, 3810-193 Aveiro, Portugal e-mail: enide@ua.pt

Luís Almeida Vieira

CMUP-Center of research of Mathematics of University of Porto, Department of Mathematics of Faculty of Sciences of University of Porto, Rua do Campo Alegre, 687; 4169-007, Porto, Portugal e-mail: 1vieira@fc.up.pt 
In this work we consider association schemes with $d$ classes and the corresponding Bose-Mesner algebra, $\mathscr{A}$, that is the algebra spanned by the matrices of the association scheme, as well as the unique basis of minimal orthogonal idempotents $\left\{E_{0}, \ldots, E_{d}\right\}$ associated to $\mathscr{A}$. We consider some special sums and products of these idempotents to prove some results over the Krein parameters of the association scheme.

This paper is organized as follows. In Section 2 the theory of association schemes is surveyed, while in Section 3 we present some important notation and matrix theory results. Then, in Section 4, we prove some results over the Krein parameters of an association scheme, namely a new upper bound for some of the Krein parameters. We finish the paper with two examples of association schemes which proves the optimality of our bound (Section 5).

\section{Association schemes and the Bose-Mesner algebra}

In this section we present relevant concepts for our work which can be seen, for instance, in [1].

An association scheme with $d$ associate classes on a finite set $X$ is a partition of $X \times X$ into sets $R_{0}, R_{1}, \ldots, R_{d}$, that are relations on $X$ such that

(i) $R_{0}=\{(x, x): x \in X\}$;

(ii) if $(x, y) \in R_{i}$, then $(y, x) \in R_{i}$, for all $x, y$ in $R_{i}$ and $i$ in $\{0,2, \ldots, d\}$;

(iii) for all $i, j, l$ in $\{0,1, \ldots, d\}$ there is an integer $p_{i j}^{l}$ such that, for all $(x, y)$ in $R_{l}$

$$
\mid\left\{z \in X:(x, z) \in R_{i} \text { and }(z, y) \in R_{j}\right\} \mid=p_{i j}^{l} .
$$

The numbers $p_{i j}^{l}$ are called the intersection numbers of the association scheme. In the case we have $(x, y) \in R_{i}$, the elements $x$ and $y$ of $X$ are called $i$-th associates. It is usual to observe the intersection numbers as the entries of the so called intersection matrices $L_{0}, L_{1}, \ldots, L_{d}$, with $\left(L_{i}\right)_{l j}=p_{i j}^{l}$, where $L_{0}=I_{n}$.

The definition presented above is due to Bose and Shimamoto, [4], and by axiom (ii) the relations $R_{i}$ are all symmetric. This is why an association scheme defined in this way is normally called symmetric. A more general definition can be seen in [6]. Along this text we will only consider symmetric association schemes.

The associate classes $R_{0}, R_{1}, \ldots, R_{d}$ of a symmetric association scheme can be described by their adjacency matrices $A_{0}, A_{1}, \ldots, A_{d}$, where each $A_{i}$ is a matrix of order $n$ defined by $\left(A_{i}\right)_{x y}=1$, if $(x, y) \in R_{i}$, and $\left(A_{i}\right)_{x y}=0$, otherwise. We also have

(a) $A_{0}=I_{n}$;

(b) $\sum_{i=0}^{d} A_{i}=J_{n}$

(c) $A_{i}=A_{i}^{\top}, \forall i \in\{0,1, \ldots, d\}$

(d) $A_{i} A_{j}=\sum_{l=0}^{d} p_{i j}^{l} A_{l}, \forall i, j \in\{0,1, \ldots, d\}$;

where $I_{n}$ and $J_{n}$ are the identity and the all ones matrices of order $n$, respectively, and $A^{T}$ denotes the transpose of $A$. Note that $(b)$ implies that the matrices $A_{i}, i=$ 
$0,1, \ldots, d$ are linearly independent. It is also well known (see [1, Lemma 1.3]) that the commutativity of the scheme asserts that $p_{i j}^{l}=p_{j i}^{l}$ and thus $A_{i} A_{j}=A_{j} A_{i}$, for all $i, j$ in $\{0,1, \ldots d\}$.

We can acknowledge $A_{1}, A_{2}, \ldots, A_{d}$ as adjacency matrices of undirected simple graphs $G_{1}, G_{2}, \ldots, G_{d}$, with common vertex set $V$. Two vertices $u$ and $v$ of $V$ are $i$-related if $u v$ is an edge in $G_{i}$, for $i$ in $\{1,2, \ldots, d\}$.

The simpler association schemes are those with only one class. It corresponds to $A_{0}=I_{n}$ and $A_{1}=J_{n}-I_{n}$. Since $G_{1}$ is the complete graph this situation is out of interest. The next simpler case regards symmetric association schemes with two classes which is equivalent to strongly regular graphs. In fact, we have $A_{0}=I_{n}$, $A_{1}, A_{2}=J_{n}-A_{1}-I_{n}$, where $A_{1}$ and $A_{2}$ correspond to the adjacency matrices of a strongly regular graph and it's complement, respectively. Conversely, if $A$ is the adjacency matrix of a strongly regular graph, then $I_{n}, A, J_{n}-A-I_{n}$ form an association scheme with two classes.

The matrices $A_{0}, A_{1}, \ldots, A_{d}$ of a symmetric association scheme generate a commutative algebra, $\mathscr{A}$, with dimension $d+1$, of symmetric matrices with constant diagonal. This algebra is called the Bose-Mesner algebra of the scheme because it was firstly studied by these two mathematicians in [3]. Note that $\mathscr{A}$ is an algebra with respect to the usual matrix product as well as to the Hadamard (or Schur) product, defined for two matrices $A, B$ of order $n$ as the componentwise product: $(A \circ B)_{i j}=A_{i j} B_{i j}$. The algebra $\mathscr{A}$ is commutative and associative relatively to this product with unit $J_{n}$.

An element $E$ in $\mathscr{A}$ is an idempotent if $E^{2}=E$. Two idempotents $E$ and $F$ in $\mathscr{A}$ are orthogonal if $E F=0$. The Bose-Mesner algebra $\mathscr{A}$ has a unique basis of minimal orthogonal idempotents $\left\{E_{0}, \ldots, E_{d}\right\}$ such that

$$
\begin{aligned}
E_{i} E_{j} & =\delta_{i j} E_{i}, \\
\sum_{i=0}^{d} E_{i} & =I_{n} .
\end{aligned}
$$

Let $\mathscr{A}$ be an association scheme with $d$ classes. If $A_{j} \in \mathscr{A}, j \in\{0,1 \ldots, d\}$ has $d+1$ distinct eigenvalues, namely $\lambda_{0}, \lambda_{1}, \ldots, \lambda_{d}$, the idempotents $E_{i}$ can be obtained as the projectors associated to the matrix $A_{j}$ through the equality:

$$
E_{i}=\prod_{l=0, l \neq i}^{d} \frac{A_{j}-\lambda_{l} I_{n}}{\lambda_{i}-\lambda_{l}}
$$

Besides the intersection numbers already introduced in the beginning of the section each association scheme contains three more families of parameters: the eigenvalues, the dual eigenvalues and the Krein parameters. In fact, there are scalars $p_{i}(j)$ and $q_{i}(j)$ such that, for all $i=0,1, \ldots, d$, we have 


$$
\begin{aligned}
A_{i} & =\sum_{j=0}^{d} p_{i}(j) E_{j} \text { and } \\
E_{i} & =\sum_{j=0}^{d} q_{i}(j) A_{j},
\end{aligned}
$$

where the numbers $p_{i}(j)$ and $q_{i}(j)$ are the eigenvalues and the dual eigenvalues of the scheme, respectively. We also define the eigenmatrix, $P=\left(P_{i j}\right)$, and the dual eigenmatrix, $Q=\left(Q_{i j}\right)$, each with dimension $(d+1) \times(d+1)$, as $P_{i j}=p_{j}(i)$ and $Q_{i j}=q_{j}(i)$, respectively. From (2) and (3) one can deduce that $P Q=I_{n}$. As a consequence, the dual eigenvalues are determined by the eigenvalues of $\mathscr{A}$.

Finally, the Krein parameters discovered by Scott, [12], of an association scheme with $d$ classes are the numbers $q_{i j}^{l}$, with $i, j, l \in\{0,1, \ldots, d\}$, such that

$$
E_{i} \circ E_{j}=\sum_{l=0}^{d} q_{i j}^{l} E_{l}
$$

These parameters can be seen as dual parameters of the intersection numbers and they are determined by the eigenvalues of the scheme. The Krein parameters of an association scheme with $d$ classes can also be considered as the entries of the matrices $L_{0}^{*}, L_{1}^{*}, \ldots, L_{d}^{*}$, such that $\left(L_{i}^{*}\right)_{l j}=q_{i j}^{l}$, which are called the dual intersection matrices of the scheme.

\section{Matrix tools}

In this section we introduce some notation and some Matrix Theory results that are used in our work in Section 4.

We denote by $\mathscr{M}_{n}(\mathbb{R})$ the space of square matrices with real entries and by $\mathscr{M}_{m, n}(\mathbb{R})$ the space of $m \times n$ matrices with real entries. The space of hermitian matrices with complex entries and dimension $n$ is denoted by $\operatorname{Herm}_{n}(\mathbb{C})$ and $\operatorname{Sym}_{n}(\mathbb{R})$ denotes the space of $n$ dimensional real symmetric matrices. Besides the Hadamard product already introduced in Section 2, we denote by $\otimes$ the Kronecker product, for matrices $C=\left[c_{i j}\right] \in \mathscr{M}_{m, n}(\mathbb{R})$ and $D=\left[d_{i j}\right] \in \mathscr{M}_{p, q}(\mathbb{R})$, defined by

$$
C \otimes D=\left(\begin{array}{ccc}
c_{11} D & \cdots & c_{1 n} D \\
\vdots & \ddots & \vdots \\
c_{m 1} D & \cdots & c_{m n} D
\end{array}\right) .
$$

The next result is of central importance in the proof of our results. For $B \in \operatorname{Sym}_{n}(\mathbb{R})$, we denote the eigenvalues of $B$ in increasing order by $\lambda_{1}(B) \leq \lambda_{2}(B) \leq \cdots \leq \lambda_{n}(B)$.

Theorem 1. [10, Eigenvalues Interlacing Theorem] $\operatorname{Let}_{A} \in \operatorname{Sym}_{n}(\mathbb{R})$ and $A_{r}$ denote any principal submatrix of $A$. Then, the eigenvalues of $A_{r}$ interlace those of $A$ in the 
sense that:

$$
\lambda_{i}(A) \leq \lambda_{i}\left(A_{r}\right) \leq \lambda_{n-r+i}(A),
$$

for each $1 \leq i \leq r$

Note that $A_{r}$ is obtained by deleting $n-r$ rows and the corresponding columns from A.

The next result shows that $A \circ B$ is a principal submatrix of $A \otimes B$. Note that $A(\alpha, \beta)$ denotes a submatrix of $A \in \mathscr{M}_{m, n}(\mathbb{R})$ determined by some index sets $\alpha$ and $\beta$.

Lemma 1. [11, Lemma 5.1.1] If $A, B \in \mathscr{M}_{m, n}(\mathbb{R})$, then

$$
A \circ B=(A \otimes B)(\alpha, \beta)
$$

in which $\alpha=\left\{1, m+2,2 m+3, \ldots, m^{2}\right\}$ and $\beta=\left\{1, n+2,2 n+3, \ldots, n^{2}\right\}$. In particular, if $m=n, A \circ B$ is a principal submatrix of $A \otimes B$.

By Lemma 1 and since the eigenvalues of $A \otimes B$ are the product between the eigenvalues of $A$ with the eigenvalues of $B$, we have the following corollary of Theorem 1, (see [11]).

Corollary 1. If $A, B \in \operatorname{Herm}_{n}(\mathbb{C})(\operatorname{Sym}(n, \mathbb{R}))$, then:

(i) $\quad \lambda_{\min }(A \circ B) \geq \lambda_{\min }(A) \lambda_{\min }(B)$;

(ii) $\quad \lambda_{\max }(A \circ B) \leq \lambda_{\max }(A) \lambda_{\max }(B)$.

\section{Some results on the Krein parameters of an association scheme}

In this section we make use of the tools presented in Section 3 to prove some results over the Krein parameters of an association scheme.

The following result establishes a formula for the calculation of the Krein parameters of an association scheme.

Proposition 1. Let $\left\{A_{0}, A_{1}, \ldots, A_{d}\right\}$ be an association scheme with $d$ classes and $j, k, l \in\{0,1, \ldots, d\}$. Then

$$
q_{j k}^{l}=\sum_{m=0}^{d} Q_{m j} Q_{m k} P_{m l},
$$

with $P$ and $Q$ the eigenmatrix and the dual eigenmatrix of the association scheme, respectively.

Proof. Let $\left\{A_{0}, A_{1}, \ldots, A_{d}\right\}$ be an association scheme with $d$ classes, $P$ and $Q$ the eigenmatrix and the dual eigenmatrix of the association scheme, respectively, and $\left\{E_{0}, E_{1}, \ldots, E_{d}\right\}$ the unique basis of minimal orthogonal idempotents of the underlying Bose-Mesner algebra $\mathscr{A}$. 
Let $j, k, l \in\{0,1, \cdots, d\}$. We have $E_{j}=\sum_{i=0}^{d} Q_{i j} A_{i}$ and $E_{k}=\sum_{i=0}^{d} Q_{i k} A_{i}$. Therefore,

$$
E_{j} \circ E_{k}=\sum_{i=0}^{d} Q_{i j} Q_{i k} A_{i}
$$

Also, we have the equality

$$
E_{j} \circ E_{k} E_{l}=\sum_{i=0}^{d} Q_{i j} Q_{i k} A_{i} E_{l} .
$$

From (2), we conclude that $A_{i} E_{l}=P_{l i}$. Thus

$$
E_{j} \circ E_{k} E_{l}=\sum_{i=0}^{d} Q_{i j} Q_{i k} P_{l i} E_{l} .
$$

Therefore

$$
q_{j k}^{l}=\sum_{i=0}^{d} Q_{i j} Q_{i k} P_{l i} .
$$

Making use of the entries of the matrices $P$ and $Q$, the formula given by equality (4) allow us to easily calculate the Krein parameters of an association scheme. Furthermore, the Krein parameters of an association scheme satisfy the following results.

Theorem 2. The Krein parameters of an association scheme with d classes satisfy the following properties.

1. For $l \in\{0,1, \ldots, d\}$ the following equality holds:

$$
\sum_{0 \leq i, j \leq d} q_{i j}^{l}=1
$$

2. For $l, r \in\{0,1, \ldots, d\}$, we have

$$
\sum_{\substack{0 \leq i \leq r-1 \\ r \leq j \leq d}} q_{i j}^{l} \leq \frac{1}{2}
$$

Proof. Let $\left\{A_{0}, A_{1}, \ldots, A_{d}\right\}$ be an association scheme with $d$ classes, $\mathscr{A}$ the underlying Bose-Mesner algebra and $\left\{E_{0}, E_{1}, \ldots, E_{d}\right\}$ the unique basis of minimal orthogonal idempotents of $\mathscr{A}$.

1. From equality

$$
\left(\sum_{i=0}^{d} E_{i}\right) \circ\left(\sum_{j=0}^{d} E_{j}\right)=I_{n},
$$

we conclude that, for $l \in\{0,1, \ldots, d\}$, 


$$
\left(\sum_{i=0}^{d} E_{i} \circ E_{j}\right) E_{l}=E_{l}
$$

from which (5) naturally arrises.

2. Let $r \in\{1,2, \ldots, d\}$ and $B$ be the following matrix

$$
\begin{aligned}
B & =\left(E_{0}+E_{1}+\cdots+E_{r-1}\right) \otimes\left(E_{r}+E_{r+1}+\cdots+E_{d}\right) \\
& +\left(E_{r}+E_{r+1}+\cdots+E_{d}\right) \otimes\left(E_{0}+E_{1}+\cdots+E_{r-1}\right) .
\end{aligned}
$$

Since $B$ is an idempotent matrix its eigenvalues belong to the set $\{0,1\}$. By Lemma 1, we observe that matrix $B$ has a principal submatrix, $C$, given by

$$
\begin{aligned}
C & =\left(E_{0}+E_{1}+\cdots+E_{r-1}\right) \circ\left(E_{r}+E_{r+1}+\cdots+E_{d}\right) \\
& +\left(E_{r}+E_{r+1}+\cdots+E_{d}\right) \circ\left(E_{0}+E_{1}+\cdots+E_{r-1}\right),
\end{aligned}
$$

and since the Hadamard product is commutative, $C$ is given simply by

$$
C=2\left(E_{0}+E_{1}+\cdots+E_{r-1}\right) \circ\left(E_{r}+E_{r+1}+\cdots+E_{d}\right) .
$$

Now, applying Theorem 1 , we conclude that, for $l \in\{0,1, \ldots, d\}$,

$$
0 \leq 2 \sum_{\substack{0 \leq i \leq r-1 \\ r \leq j \leq d}} q_{i j}^{l} \leq 1
$$

and inequality (6) follows imediatly.

The following result is a consequence of Theorem 2 .

Corollary 2. For each $l \in\{0,1, \ldots, d\}$, the Krein parameters of an association scheme with d classes satisfy the following properties:

1. $\sum_{i=0}^{d} q_{i i}^{l} \leq 1$;

2. $\min _{i \in\{0, \ldots, d\}}\left\{q_{i i}^{l}\right\} \leq \frac{1}{d+1}$.

Our last result establishes a new upper bound for some of the Krein parameters of an association scheme.

Theorem 3. If $l, i, j \in\{0,1, \ldots, d\}, i \neq j$, then

$$
q_{i j}^{l} \leq \frac{1}{2}
$$

Furthermore, if it exists an $i \in\{0,1, \ldots, d\}, i \neq j$, such that $q_{i i}^{l} \neq 0$, then the inequality presented is strict. 
Proof. Let $\left\{A_{0}, A_{1}, \ldots, A_{d}\right\}$ be an association scheme with $d$ classes, $\mathscr{A}$ the underlying Bose-Mesner algebra and $\left\{E_{0}, E_{1}, \ldots, E_{d}\right\}$ the unique basis of minimal orthogonal idempotents of $\mathscr{A}$.

Let $i, j \in\{0,1, \ldots, d\}$. The matrix

$$
B=\sum_{\substack{0 \leq r \leq d \\ r \neq j}}\left(E_{r} \otimes E_{r}\right)+E_{i} \otimes E_{j}+E_{j} \otimes E_{i}
$$

is an idempotent matrix which possesses a principal submatrix, $C$, given by

$$
C=\sum_{\substack{0 \leq r \leq d \\ r \neq j}}\left(E_{r} \circ E_{r}\right)+E_{i} \circ E_{j}+E_{j} \circ E_{i},
$$

(see Lemma 1). For each $l \in\{0,1, \ldots, d\}$ we also have that

$$
\begin{aligned}
& {\left[\sum_{\substack{0 \leq r \leq d \\
r \neq j}}\left(E_{r} \circ E_{r}\right)+E_{i} \circ E_{j}+E_{j} \circ E_{i}\right] E_{l} } \\
= & \sum_{\substack{0 \leq r \leq d \\
r \neq j}}\left[\left(E_{r} \circ E_{r}\right) E_{l}\right]+\left(E_{i} \circ E_{j}\right) E_{l}+\left(E_{j} \circ E_{i}\right) E_{l} \\
= & \sum_{\substack{0 \leq r \leq d \\
r \neq j}}\left(q_{r r}^{l} E_{l}\right)+q_{i j}^{l} E_{l}+q_{i j}^{l} E_{l} .
\end{aligned}
$$

Then, by Theorem 1, the eigenvalues of $C$ are bounded by 0 and 1 and therefore, for each $l \in\{0,1, \ldots, d\}$ we have

$$
0 \leq \sum_{\substack{0 \leq r \leq d \\ r \neq j}}\left(q_{r r}^{l}\right)+2 q_{i j}^{l} \leq 1 .
$$

By property (1.) of Corollary 2, from (7), we conclude the statements of Theorem 3.

\section{Some examples}

In this section we present two examples for our upper bound of the Krein parameters of an association scheme. The first example is based on the notation presented in the paper [5].

Example 1. Let $n$ be an even natural number and $U_{i, j} \in \mathscr{M}_{n}(\mathbb{R})$ be the matrices defined by $\left(U_{i, j}\right)_{p q}=\delta_{i p} \delta_{j q}$, for $i, j, p, q \in\{1,2, \ldots, n\}$. Let $m=\frac{n}{2}+1$. Now we consider the family of matrices $\mathscr{F}=\left\{B_{i}\right\}_{i \in\{1, \ldots, m\}}$ such that: 
- $B_{1}=I_{n}$

- $B_{r}=\sum_{l=r}^{n} U_{l, l-r+1}+\sum_{l=r}^{n} U_{l-r+1, l}+\sum_{l=1}^{r-1} U_{n-r+1+l, l}+\sum_{l=1}^{r-1} U_{l, n-r+1+l}, r=2, \ldots, m$;

- $B_{m}=\sum_{l=1}^{m-1} U_{n-m+1+l, l}+\sum_{l=1}^{m-1} U_{l, n-m+1+l}$.

From the definition, the matrices $A_{j}, j \in\{2, \ldots, m\}$ are symmetric matrices and zero diagonal elements.

For $i=0,1, \ldots, n-1$, the matrices $C_{i}$ are defined by the formula

$$
\left(C_{i}\right)_{p q}=\left\{\begin{array}{l}
1 \text { if } q=p \oplus_{n} i, \\
0 \text { if } q \neq p \oplus_{n} i,
\end{array},\right.
$$

where $\oplus_{n}$ denotes the sum modulo $n$. Then we have that the matrices $B_{j}, j \in$ $\{2, \ldots, m\}$ are given by:

$$
\begin{aligned}
B_{1} & =C_{0} \\
B_{j} & =C_{j-1}+C_{n}-(j-1), j \in\{2, \ldots, m-1\} \\
B_{m} & =C_{m-1} .
\end{aligned}
$$

Since the matrices $C_{i}$ are commutative, then the family $\mathscr{F}$ is a commutative matrices family.

Now we construct the following association scheme with two classes $\mathscr{A}=$ $\left\{A_{0}, A_{1}, A_{2}\right\}$ where:

$$
\begin{aligned}
& A_{0}=I_{n} \\
& A_{1}=\sum_{i=2}^{m-1} B_{i} \\
& A_{2}=J_{n}-A_{1}-I_{n},
\end{aligned}
$$

where $J_{n}$ is the all ones matrix. The minimal polynomial of $A_{1}$ is given by

$$
p(\lambda)=\lambda(\lambda+2)(\lambda-n+2) .
$$

The unique basis of minimal idempotents of $\mathscr{A}$ is the set $\left\{E_{0}, E_{1}, E_{2}\right\}$ such that

$$
\begin{aligned}
& E_{0}=\frac{1}{n} J_{n}, \\
& E_{1}=\frac{1}{2} I_{n}-\frac{1}{2}\left(J_{n}-A-I_{n}\right), \\
& E_{2}=\frac{n-2}{2 n} I_{n}-\frac{1}{n} A+\frac{n-2}{2 n}\left(J_{n}-A-I_{n}\right) .
\end{aligned}
$$

Then the Krein parameter $q_{12}^{1}$ can be written as

$$
q_{12}^{1}=\frac{n-2}{2 n},
$$

which converges to $1 / 2$ when $n$ tends to infinity. 
Since the association schemes of two classes are particular cases of association schemes, we may conclude, from Example 1 that the upper bound $1 / 2$ for the Krein parameters $q_{i j}^{l}$, for $i \neq j$, in Theorem 3, is optimal for an association scheme with any number of classes.

In our final example we present a family of association schemes with three classes constructed from symmetric designs. This family has an infinite number of elements and is presented and studied in [13].

Let $\mathscr{P}$ be a set of points and $\mathscr{B}$ be a set of blocks, where a block is a subset of $\mathscr{P}$. Then, the ordered pair $(\mathscr{P}, \mathscr{B})$ is a symmetric design with parameters $(n, k, c)$ if it satisfies the following properties:

(i) $\mathscr{B}$ is a subset of the power set of $\mathscr{P}$;

(ii) $|\mathscr{P}|=|\mathscr{B}|=n$;

(iii) $\forall b \in \mathscr{B},|b|=k$;

(iv) $\forall p \in \mathscr{P},|\{b \in \mathscr{B}: p \in b\}|=k$;

(v) $\forall p_{1}, p_{2} \in \mathscr{P}, p_{1} \neq p_{2},\left|\left\{b \in \mathscr{B}: p_{1}, p_{2} \in b\right\}\right|=c$;

(vi) $\forall b_{1}, b_{2} \in \mathscr{B}, b_{1} \neq b_{2},\left|\left\{p \in \mathscr{P}: p \in b_{1} \wedge p \in b_{2}\right\}\right|=c$.

Example 2. Given a symmetric design with parameters $(n, k, c)$, we build a three class association scheme, as in [13], in the following manner. Let $X=\mathscr{P} \cup \mathscr{B}$. We define the following relations in $X \times X$ :

$$
\begin{aligned}
& R_{0}=\{(x, x): x \in X\} ; \\
& R_{1}=\{(x, y) \in \mathscr{P} \times \mathscr{B}: x \in y\} \cup\{(y, x) \in \mathscr{B} \times \mathscr{P}: x \in y\} ; \\
& R_{2}=\{(x, y) \in \mathscr{P} \times \mathscr{P}: x \neq y\} \cup\{(x, y) \in \mathscr{B} \times \mathscr{B}: x \neq y\} ; \\
& R_{3}=\{(x, y) \in \mathscr{P} \times \mathscr{B}: x \notin y\} \cup\{(y, x) \in \mathscr{B} \times \mathscr{P}: x \notin y\} .
\end{aligned}
$$

Through the axioms $(i)-(v i)$ of a symmetric design it is proved that $R_{0}, R_{1}, R_{2}, R_{3}$ constitute an association scheme with three classes over $X$. From the relations above we compute the intersection matrices of the association scheme, given by $L_{0}=I_{4}$,

$$
\begin{aligned}
L_{1} & =\left(\begin{array}{lllc}
0 & k & 0 & 0 \\
1 & 0 & k-1 & 0 \\
0 & c & 0 & k-c \\
0 & 0 & k & 0
\end{array}\right), \quad L_{2}=\left(\begin{array}{cccc}
0 & 0 & n-1 & 0 \\
0 & k-1 & 0 & n-k \\
1 & 0 & n-2 & 0 \\
0 & k & 0 & n-k-1
\end{array}\right), \\
L_{3} & =\left(\begin{array}{cccc}
0 & 0 & 0 & n-k \\
0 & 0 & n-k & 0 \\
0 & k-c & 0 & n-2 k+c \\
1 & 0 & n-k-1 & 0
\end{array}\right)
\end{aligned}
$$

Now, using axioms $(a)-(d)$ of the matrices of the Bose-Mesner algebra, $\mathscr{A}=$ $\left\{A_{0}, A_{1}, A_{2}, A_{3}\right\}$, we obtain their multiplication table. 


\begin{tabular}{|c|c|c|c|c|}
\hline$\times$ & $A_{0}$ & $A_{1}$ & $A_{2}$ & $A_{3}$ \\
\hline$A_{0}$ & $A_{0}$ & $A_{1}$ & $A_{2}$ & $A_{3}$ \\
\hline$A_{1}$ & $A_{1}$ & $k A_{0}+c A_{2}$ & $(k-1) A_{1}+k A_{3}$ & $(k-c) A_{2}$ \\
\hline$A_{2}$ & $A_{2}$ & $(k-1) A_{1}+k A_{3}$ & $(n-1) A_{0}+(n-2) A_{2}$ & $(n-k) A_{1}+(n-k-1) A_{3}$ \\
\hline$A_{3}$ & $A_{3}$ & $(k-c) A_{2}$ & $(n-k) A_{1}+(n-k-1) A_{3}$ & $(n-k) A_{0}+(n-2 k+c) A_{2}$ \\
\hline
\end{tabular}

Making use of the multiplication table of the matrices of $\mathscr{A}$, we can calculate the powers of $A_{1}$ to obtain the minimal polynomial of $A_{1}$, which is given by:

$$
p_{A_{1}}(\lambda)=\lambda^{4}+\left(-k^{2}-k+c\right) \lambda^{2}+k^{2}(k-c) .
$$

Applying formula (1) in order to matrix $A_{1}$ and considering the eigenvalues of the polynomial (8), $\lambda_{0}=k, \lambda_{1}=-k, \lambda_{2}=\sqrt{k-c}$ and $\lambda_{3}=-\sqrt{k-c}$, we obtain the elements of the unique basis of minimal orthogonal idempotents of $\mathscr{A}$ :

$$
\begin{aligned}
& E_{0}=\frac{A_{0}+A_{1}+A_{2}+A_{3}}{2 n}=\frac{J_{n}}{2 n} \\
& E_{1}=\frac{A_{0}-A_{1}+A_{2}-A_{3}}{2 n} \\
& E_{2}=\frac{(n-1) \sqrt{k-c} A_{0}+(n-k) A_{1}-\sqrt{k-c} A_{2}-k A_{3}}{2 n \sqrt{k-c}} \\
& E_{3}=\frac{(n-1) \sqrt{k-c} A_{0}-(n-k) A_{1}-\sqrt{k-c} A_{2}+k A_{3}}{2 n \sqrt{k-c}} .
\end{aligned}
$$

Now we apply equalities (2) and (3) to compute the matrices $P$ and $Q$, respectively:

$$
P=\left(\begin{array}{cccc}
1 & k & n-1 & n-k \\
1 & -k & n-1 & k-n \\
1 & \sqrt{k-c} & -1 & -\sqrt{k-c} \\
1 & -\sqrt{k-c} & -1 & \sqrt{k-c}
\end{array}\right), \quad Q=\frac{1}{2 n}\left(\begin{array}{cccc}
1 & 1 & n-1 & n-1 \\
1 & -1 & -\frac{k-n}{\sqrt{k-c}} & \frac{k-n}{\sqrt{k-c}} \\
1 & 1 & -1 & -1 \\
1 & -1 & -\frac{k}{\sqrt{k-c}} & \frac{k}{\sqrt{k-c}}
\end{array}\right)
$$

Finally, we obtain the dual intersection matrices of this association scheme by applying formula (4) from Proposition 1: $L_{0}^{*}=I_{4} / 2 n$, 


$$
\begin{aligned}
& L_{1}^{*}=\frac{1}{2 n}\left(\begin{array}{llll}
0 & 1 & 0 & 0 \\
1 & 0 & 0 & 0 \\
0 & 0 & 0 & 1 \\
0 & 0 & 1 & 0
\end{array}\right) \\
& L_{2}^{*}=\frac{1}{2 n}\left(\begin{array}{cccc}
0 & 0 & n-1 & 0 \\
0 & 0 & 0 & n-1 \\
1 & 0 & \frac{n-2}{2}-\frac{n-2 k}{2 \sqrt{k-c}} & \frac{n-2}{2}+\frac{n-2 k}{2 \sqrt{k-c}} \\
0 & 1 & \frac{n-2}{2}+\frac{n-2 k}{2 \sqrt{k-c}} & \frac{n-2}{2}-\frac{n-2 k}{2 \sqrt{k-c}}
\end{array}\right), \\
& L_{3}^{*}=\frac{1}{2 n}\left(\begin{array}{cccc}
0 & 0 & 0 & n-1 \\
0 & 0 & n-1 & 0 \\
0 & 1 & \frac{n-2}{2}+\frac{n-2 k}{2 \sqrt{k-c}} & \frac{n-2}{2}-\frac{n-2 k}{2 \sqrt{k-c}} \\
1 & 0 & \frac{n-2}{2}-\frac{n-2 k}{2 \sqrt{k-c}} & \frac{n-2}{2}+\frac{n-2 k}{2 \sqrt{k-c}}
\end{array}\right) .
\end{aligned}
$$

From the dual intersection matrices presented above, it is possible to extract some evidence of the optimality of the upper bound $1 / 2$, for the Krein parameters $q_{i j}^{l}$, with $i \neq j$, presented in Theorem 3 . In fact, we can observe that

$$
q_{23}^{0}=\left(L_{2}^{*}\right)_{03}=\frac{n-1}{2 n}
$$

and this value converges to $1 / 2$, when $n$ tends to infinity.

With these two examples we show that the upper bound presented in Theorem 3 , for the Krein parameters $q_{i j}^{l}$, with $i \neq j$ of any association scheme, is optimal and cannot be improved in the general case.

Acknowledgements 1. Enide Andrade Martins is supported in part by FEDER funds through COMPETE Operational Programme Factors of Competitiveness ("Programa Operacional Factores de Competitividade") and by Portuguese funds through the Center for Research and Develpment in Mathematics and Applications and the Portuguese Foundation for Science and Technology ("FCT - Fundação para a Ciência e a Tecnologia") within project PEestC/MAT/UI4106/2011 with COMPETE number FCOMP-01-0124-FEDER-022690 and Project PTDC/MAT/ 112276/2009.

2. Luís Vieira research funded by the European Regional Development Fund through the program COMPETE and by the Portuguese Government through the Center of Mathematics of University of Porto and the Portuguese Foundation for Science and Technology ("FCT - Fundação para a Ciência e a Tecnologia") under the project PEest-C/MAT/UI0144/2011.

\section{References}

1. Bailey, R. A.: Association Schemes, Designed Experiments, Algebra and Combinatorics, Cambridge University Press, Cambridge (2004)

2. Bose, R. C.: Strongly regular graphs, partial geometries and partially balanced designs, Pacific J. Math 13, 389-419 (1963)

3. Bose, R. C., Mesner, D. M.: On linear associative algebras corresponding to association schemes of partially balanced designs, Ann. Math. Statist. 30, 21-38 (1959) 
4. Bose, R. C., Shimamoto, T.: Classification and analysis of partially balanced incomplete block designs with two associate classes, J. Am. Statist. Assoc. 47, 151-184 (1952)

5. Cardoso, D. M., Vieira, L. A.: Euclidean Jordan algebras with strongly regular graphs, Journal of Mathematical Sciences 120, 1, 881-894 (2004)

6. Delsarte, Ph.: An algebraic approach to the association schemes of coding theory, Philips Res. Rep. Suppl. 10 (1973)

7. Hanaki, A.: Character of association schemes and normal closed subsets, Graphs Combin. 19 (3), 363-369 (2003)

8. Higman, D. G.: Coherent configurations part I: Ordinary representation theory, Geometriae 4, 1-32 (1975)

9. Higman, D. G.: Coherent configurations part II: Weights, Geometriae 5, 413-424 (1976)

10. Horn, R., Johnson, C. R.: Matrix Analysis, Cambridge University Press, Cambridge (1985)

11. Horn, R., Johnson, C. R.: Topics in Matrix Analysis, Cambridge University Press, Cambidge (1991)

12. Scott, Jr. L. L.: A condition on Higman's parameters, Notices of Amer. Math. Soc. 20 A-97, 721-20-45 (1973)

13. Shakan, G., Xin, Y.: Q-Polynomial Association Schemes with Irrational Eigenvalues, A Major Qualifying Project submitted to the Faculty of the Worcester Polytechnic Institute (2012) 